\title{
On the Mitigation of RIN Transfer and Transmission Performance Improvement in Bidirectional Distributed Raman Amplifiers
}

\author{
Md Asif Iqbal, Mingming Tan, and Paul Harper
}

\begin{abstract}
We develop a novel broadband first order Raman pump for use as a forward pump in transmission experiments. Our results show significant signal relative intensity noise (RIN) reduction, to a level comparable to backward only pumping. The corresponding optical signal to noise ratio can be improved in dual and first order forward pumped $83.32 \mathrm{~km}$ bidirectional distributed Raman amplifiers by using the proposed broadband pump as a first order pump. A detailed experimental characterization of RIN, signal power evolution and performance of a $10 \times 120 \mathrm{~Gb} / \mathrm{s}$ DP-QPSK coherent WDM transmission system are presented. We report $\sim 10 \mathrm{~dB}$ RIN reduction and $0.7 \mathrm{~dB} Q$ factor improvement which allows a $1250 \mathrm{~km}$ transmission distance increase compared with conventional low RIN and narrowband $1^{\text {st }}$ order pump sources. We also demonstrate that, bidirectional pumping with only broadband $1^{\text {st }}$ order forward pumping at 50mW shows the lowest RIN transfer from pump to signal. This extends the transmission reach up to $8332 \mathrm{~km}$ with maximum distances increased by $1250 \mathrm{~km}$ and $1667 \mathrm{~km}$ compared with conventional backward only and $1^{\text {st }}$ order semiconductor forward pumped bidirectional pumping respectively.
\end{abstract}

Index Terms - Optical fibre, nonlinear effects, optical fibre communication, optical amplifiers.

\section{INTRODUCTION}

$\mathrm{D}$ ISTRIBUTED Raman amplifier (DRA) has many advantages in modern long-haul coherent dense wavelength division multiplexed (DWDM) transmission systems such as improved optical signal to noise ratio (OSNR) essential for higher order and spectrally efficient modulation formats [1], [2], flexible signal power profile along transmission span enabling efficient nonlinear compensation techniques i.e. optical phase conjugation (OPC) [3], [4] or nonlinear Fourier transform (NFT) [5]. Although DRA with bidirectional higher order Raman pumping [6], [7] is the best choice to maintain a quasi-lossless signal power profile with maximum reduction of amplified spontaneous emission (ASE)

This work was supported by FP7 ITN Programme ICONE (608099) and UK EPSRC Programme Grant UNLOC (EP/J017582/1) and PEACE (EP/L000091/1). (Corresponding author: Md Asif Iqbal)

Md Asif Iqbal, M. Tan and P. Harper are with Aston Institute of Photonic Technologies, Aston University, Birmingham B4 7ET, UK (e-mail: iqbalm7@aston.ac.uk; m.tan1@aston.ac.uk; p.harper@aston.ac.uk).

Original data for this work is available through Aston Research Explorer (https://doi.org/10.17036/researchdata.aston.ac.uk.00000340).

Copyright (c) 2015 IEEE. Personal use of this material is permitted. However, permission to use this material for any other purposes must be obtained from the IEEE by sending a request to pubs-permissions@ieee.org. noise compared with conventional backward only pumping, RIN transfer [8], [9] from forward pump to the signal is a major drawback in these systems, which may counteract the benefit of improved OSNR. The stochastic intensity fluctuations in the Raman pump also induce relative phase noise through pump-signal cross phase modulation [10] and cross-polarization interactions in polarization division multiplexed coherent transmission systems. RIN transfer mainly depends on the amplitude noise level in the pump(s), pumping configurations and chromatic dispersion of the fibre which determine the walk-off between the pump and signal. There have already been many efforts in reducing the pump to signal RIN transfer such as: introducing high dispersion fibre [11], intensity modulation in dual order Raman pumping [9], forward pumping with incoherent broadband pump [12], [13] and dual order forward pumping with optimized low reflectivity fibre Bragg grating (FBG) at $1^{\text {st }}$ order pump wavelength [14]. Recently we have proposed a RIN mitigation technique in a random distributed feedback (DFB) lasing based bidirectional, dual order DRA without any $1^{\text {st }}$ order forward seed [15]. Although this technique shows improved transmission performance, it requires very high $2^{\text {nd }}$ order forward pump power to transfer the gain efficiently to the signals which are two Stokes shift away from the pump. Using a low RIN, narrowband conventional $1^{\text {st }}$ order forward pump seed (i.e. semiconductor laser diode) improves the overall pump efficiency but transmission performance may still be limited by the RIN transfer from high power $2^{\text {nd }}$ order pump.

RIN transfer from higher order pump to signal not only depends on the pump RIN of the $1^{\text {st }}$ order seed but also on the spectral properties. Recently the use of a large bandwidth pseudo-incoherent pump for RIN transfer reduction has been demonstrated theoretically [12] showing the reduced transfer of amplitude noise due to the non-degenerate four wave mixing (FWM) process among broadband pump and signal frequencies with random phase variations. However, to the best of our knowledge, long-haul WDM coherent transmission performance using a RIN mitigated bidirectional distributed DRA with broadband forward pump is not well documented. An experimental demonstration has been reported for a single channel coherent transmission system, but the performance benefits were not clearly established with conventional backward only pumping and other pump sources [13].

We recently reported significant signal RIN suppression 
using a smooth profile broadband $1^{\text {st }}$ order forward pump, compared with conventional narrowband pump sources in dual order bidirectional DRA [16]. We have also reported some transmission performance benefits compared with conventional pumping schemes in a long-haul coherent WDM transmission setup using both dual order [17] and $1^{\text {st }}$ order [18] forward pumped bidirectional DRAs. Here we extend the results from our previous reports and demonstrate in detail that, our proposed $1^{\text {st }}$ order broadband source can be used both in dual order and $1^{\text {st }}$ order only forward pumped DRA schemes for substantial $(>10 \mathrm{~dB})$ signal RIN reduction and transmission reach extension compared with widely deployed low RIN, narrowband semiconductor pump and conventional dual order backward only pumping. Firstly, we report the details of the generation process of our proposed inherently depolarised broadband $1^{\text {st }}$ order source, which has much wider $3 \mathrm{~dB}$ bandwidth $(\sim 18 \mathrm{~nm})$ than conventional pump sources. Then, we optimize the required forward powers of $2^{\text {nd }}$ order $1365 \mathrm{~nm}$ and $1^{\text {st }}$ order broadband pumps in dual and first order forward pumping respectively, in order to determine the best balance between improved signal power distribution and minimum signal RIN performance which provides the maximum transmission performances in respective schemes. Finally, at optimum forward pump powers, coherent WDM transmission performances are compared with conventional pumping schemes to demonstrate the benefit of suppressed signal RIN using low noise DRAs. In $10 \times 120 \mathrm{~Gb} / \mathrm{s}$ DP-QPSK coherent WDM transmission system, our proposed dual order forward pumped DRA demonstrates $1250 \mathrm{~km}$ transmission reach enhancement compared with narrowband $1^{\text {st }}$ order semiconductor pumped dual order forward pumping scheme.

We also demonstrate that, bidirectional pumping with only $1^{\text {st }}$ order broadband forward pump performs better than that of dual order forward pumped bidirectional DRA for superior RIN mitigation and extends the transmission reach of similar WDM system up to $8332 \mathrm{~km}$. We also report maximum transmission reach extensions up to $1250 \mathrm{~km}$ and $1667 \mathrm{~km}$ compared with conventional dual order backward only and $1^{\text {st }}$ order semiconductor forward pumped bidirectional pumping schemes, using only $50 \mathrm{~mW}$ of optimized broadband pump power without requiring higher order pumping schemes.

\section{EXPERIMENTAL SETUP}

The configuration of dual forward pumped bidirectional DRA are shown in Fig. 1. An $83.32 \mathrm{~km}$ of standard single mode fibre (SSMF) is used as the amplifier span. The backward dual order pumping consists of a $2^{\text {nd }}$ order $1365 \mathrm{~nm}$ pump and a high reflectivity (95\%) fibre Bragg grating (FBG) with $0.6 \mathrm{~nm} 3 \mathrm{~dB}$ bandwidth and $1455 \mathrm{~nm}$ centre wavelength, placed at the end of the span to provide feedback at the $1^{\text {st }}$ order pumping wavelength [15]. We have chosen second order backward only pumping as a reference for minimum RIN DRA scheme here because of its simplicity using one backward pump, and improved noise/transmission performance due to more uniform signal power distribution compared with conventional $1^{\text {st }}$ order semiconductor laser diode pumped counterpart [15]. Dual order forward pumping consisted of a high RIN $(-113 \mathrm{~dB} / \mathrm{Hz}) 2^{\text {nd }}$ order $1365 \mathrm{~nm}$ pump combined with the generated broadband $1^{\text {st }}$ order pump at $1455 \mathrm{~nm}$, whereas only the broadband $1455 \mathrm{~nm}$ pump is used in the $1^{\text {st }}$ order forward pumping as described in section IV.

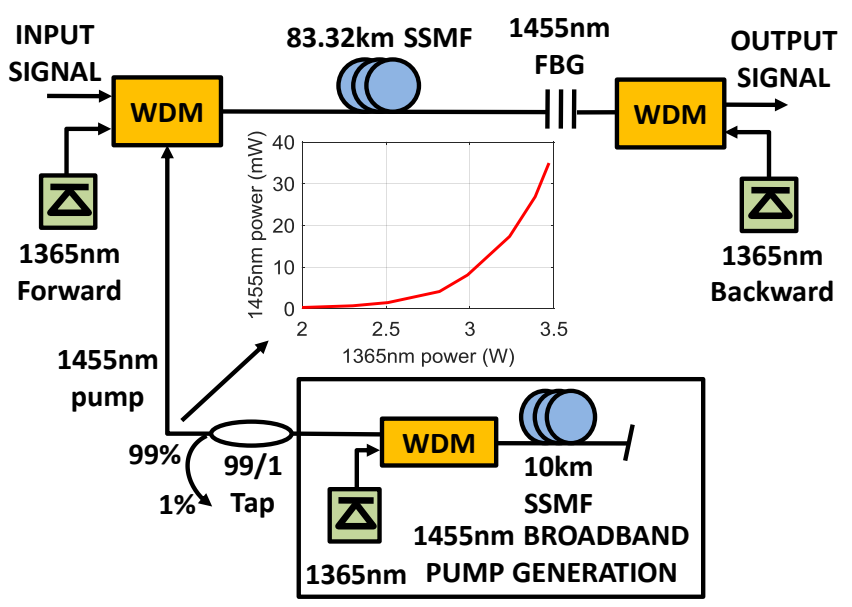

Fig. 1. Bidirectional distributed Raman amplifier span with 83.32km SSMF and proposed dual forward pumping scheme (Insert: relationship between the powers of generated broadband pump and $1365 \mathrm{~nm}$ backward pump).

In Fig.1, the broadband $1^{\text {st }}$ order forward pump seed is generated by backward pumping a $10 \mathrm{~km}$ SSMF with a separate $1365 \mathrm{~nm}$ pump laser at $3 \sim 4 \mathrm{~W}$ power as shown inside the solid box. The choice of 10km SSMF gave the maximum stable output power of the broadband pump before the onset of stochastic pulses due to excessive Rayleigh scattering feedback induced higher order stimulated Brillouin scattering (SBS) Stokes waves [19], using the available $(\sim 4 \mathrm{~W})$ power of $1365 \mathrm{~nm}$ pump. However further theoretical optimisation can be done to find the optimum balance between the SSMF length and $1365 \mathrm{~nm}$ pump power. The Stokes shifted broadband light around $1455 \mathrm{~nm}$ is first generated by Raman scattering then some portion of broadband light is Rayleigh scattered along the $10 \mathrm{~km}$ SSMF and amplified by the same $1365 \mathrm{~nm}$ pump. In a separate $10 \mathrm{~km}$ SSMF section, maximum $1.5 \mathrm{~mW}$ broadband $1455 \mathrm{~nm}$ pump power can be obtained by using $\sim 3.5 \mathrm{~W} 1365 \mathrm{~nm}$ backward pump power before the onset of stochastic parasitic lasing. Here in Fig. 1, the generated broadband $1455 \mathrm{~nm}$ pump is coupled into the main $83.32 \mathrm{~km}$ amplifier span through a $3 \times 1$ bidirectional input WDM coupler. The broadband $1455 \mathrm{~nm}$ pump also gets amplified in the main DRA span by the $1365 \mathrm{~nm}$ pump(s). Then part of the amplified $1455 \mathrm{~nm}$ light are reflected back into the seed generation (10km SSMF) span and gets further amplified by the local broadband seed generating $1365 \mathrm{~nm}$ pump. Finally, $20 \mathrm{~mW}$ broadband pump power is maintained into the DRA span and monitored using a 99/1 tap, by using optimized $1365 \mathrm{~nm}$ pump power of $\sim 3.25 \mathrm{~W}$. The relationship between different broadband pump powers into the span and generating $1365 \mathrm{~nm}$ backward pump powers is shown in Fig. 1 insert.

The proposed method is simpler and different from the previously reported techniques [13], [20], which has similar spectral and RIN profiles. Broadband pump source at any specific wavelength band can be generated using the proposed 
scheme, which requires a piece of passive fibre and high power backward Raman pumping. Whereas, generating wavelength tuned high power and low RIN broadband pump source using super luminescent diode (SLD) or ASE from semiconductor optical amplifier (SOA) is somehow difficult and requires critical design methodologies.
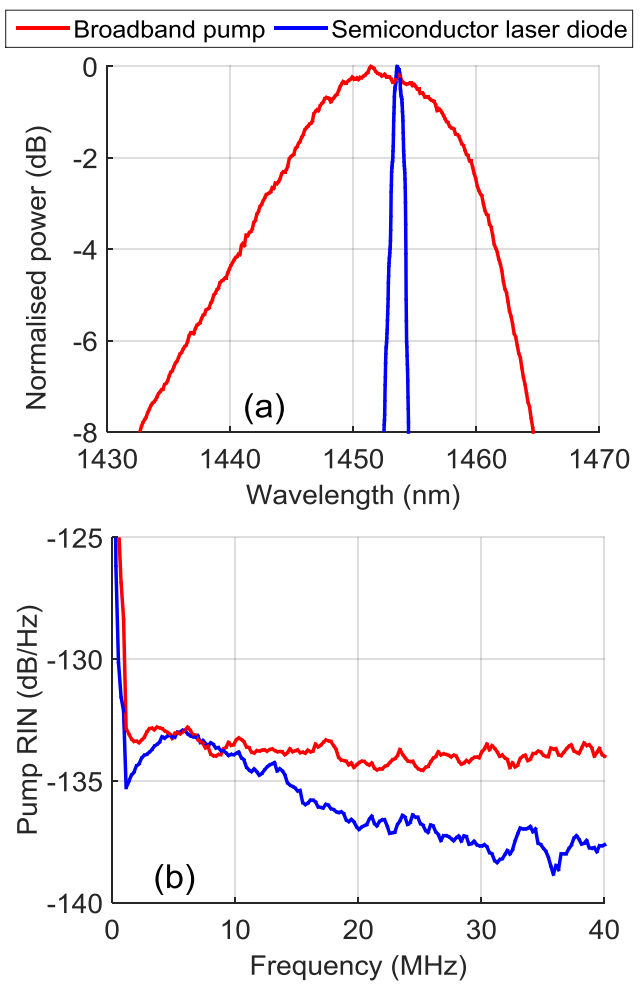

Fig. 2. Comparison of (a) first order pump spectra and (b) pump RIN between proposed broadband pump and conventional semiconductor laser diode pump.

The broadband pump seed is also inherently depolarized due to the randomness of Rayleigh scattered light. The $3 \mathrm{~dB}$ bandwidth is $\sim 18 \mathrm{~nm}$ which is much wider than the commercial semiconductor laser diode (3dB bandwidth $0.8 \mathrm{~nm}$ and RIN level $-135 \mathrm{~dB} / \mathrm{Hz}$ ). An angle connector is used at the end of $10 \mathrm{~km}$ SSMF span to minimize any reflection in order to generate a very low RIN ( -132dB/Hz) broadband pump in an open cavity based configuration [21]. The comparison of
RIN and spectral properties between different pumps are shown in Fig. 2 (a) and (b).

A coherent transmission experiment was performed in a recirculating loop setup as shown in Fig. 3. Ten DFB lasers from $194.3 \mathrm{THz}(1542.94 \mathrm{~nm})$ to $193.4 \mathrm{THz}(1550.12 \mathrm{~nm})$ with $100 \mathrm{GHz}$ spacing were multiplexed using an arrayed waveguide grating (AWG) to form the WDM grid. The output of the multiplexed signal was then combined with a $100 \mathrm{kHz}$ linewidth (LW) external cavity laser (ECL) used as "channels under test (CUT)". During each measurement, the particular DFB laser was switched off and replaced by the CUT. The continuous wave $(\mathrm{CW})$ signal channels were then QPSK modulated using a Mach-Zehnder I-Q modulator. The applied electrical signal from a pulse pattern generator (PPG) was $30 \mathrm{~Gb} / \mathrm{s}, 2^{31}-1$ word length, normal and inverse pseudo random binary sequences (PRBS) patterns with a relative delay of 18bits. The output 10×30GBaud QPSK signals were then amplified using a polarization maintaining erbium doped fibre amplifier (EDFA) and polarization multiplexed through a polarization multiplexing (POLMUX) emulator with a relative delay equivalent to 300 symbols ( 2ns) between the two polarization states to generate $10 \times 120 \mathrm{~Gb} / \mathrm{s}$ DP-QPSK signals at the input of the loop.

The transmission span in the recirculating loop was formed by the distributed Raman span with 83.32km SSMF with a total loss of $17.6 \mathrm{~dB}$ including $16.5 \mathrm{~dB}$ span loss and $1.1 \mathrm{~dB}$ passive component loss from pump/signal combiners. A dualstage EDFA was used to compensate the additional 12dB loop losses from gain flattening filter (GFF), $3 \mathrm{~dB}$ coupler and acousto-optic modulator (AOM). At the receiver, the received signal was first de-multiplexed using a tuneable bandpass filter and then amplified using an EDFA before passing it to a standard polarization diverse coherent receiver with $80 \mathrm{GSa} / \mathrm{s}$ and $36 \mathrm{GHz}$ bandwidth oscilloscope. Digital signal processing (DSP) was applied in offline post-processing for linear impairment mitigation and signal recovery. Q factors were measured from actual bit error counting and averaged over 2 million bits. A HD-FEC limit of $8.5 \mathrm{~dB} Q$ factor was considered for performance measurement.

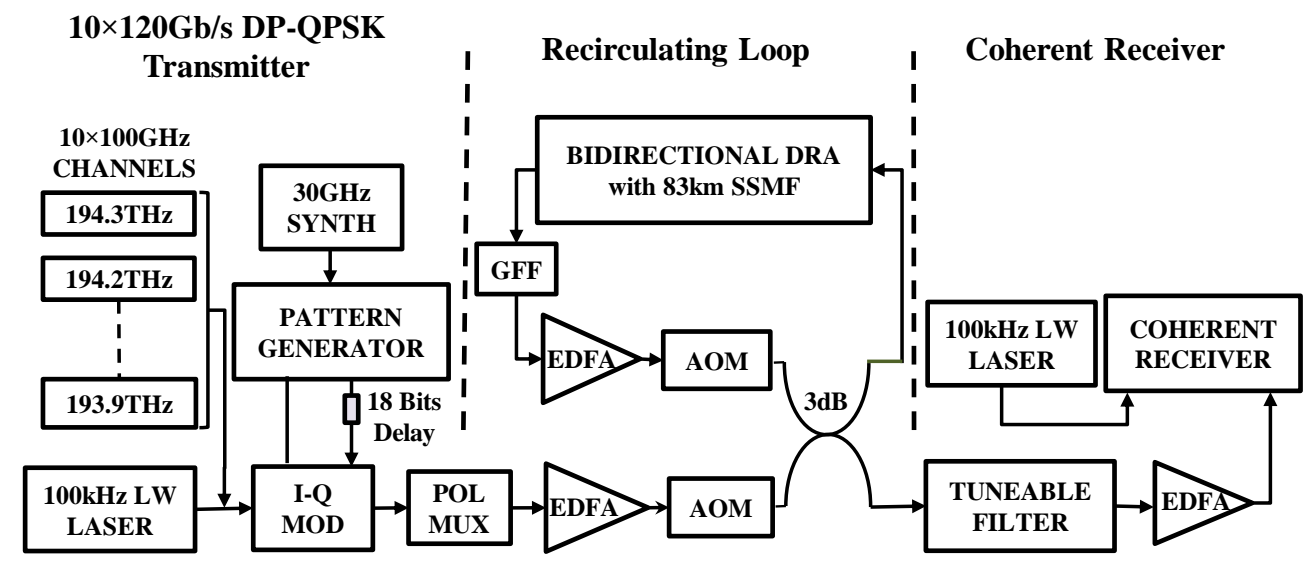

Fig. 3. Schematic diagram of long-haul coherent WDM transmission system in a recirculating loop setup. Abbreviations: SYNTH = synthesizer, POLMUX = polarization multiplexer, $\mathrm{LW}=$ linewidth, $\mathrm{GFF}=$ gain flattening filter and $\mathrm{AOM}=$ acousto-optic modulator . 


\section{RIN Mitigation With DuAL ORder ForWARD PUMPING}

In dual order forward Raman pumping, stochastic amplitude fluctuations from high power and high RIN $2^{\text {nd }}$ order pump is transferred and distributed over the random phases of the broadband $1^{\text {st }}$ order seed. The intensity noise evolution is averaged out over the wide bandwidth of the low RIN pump and subsequent RIN transfer to the signal is mitigated. In this section at first, we experimentally characterize the signal RIN and power variation along the amplifier span at different forward $1365 \mathrm{~nm}$ pump powers. Transmission performances are then compared to optimize the forward $1365 \mathrm{~nm}$ pump powers required for the best transmission results.
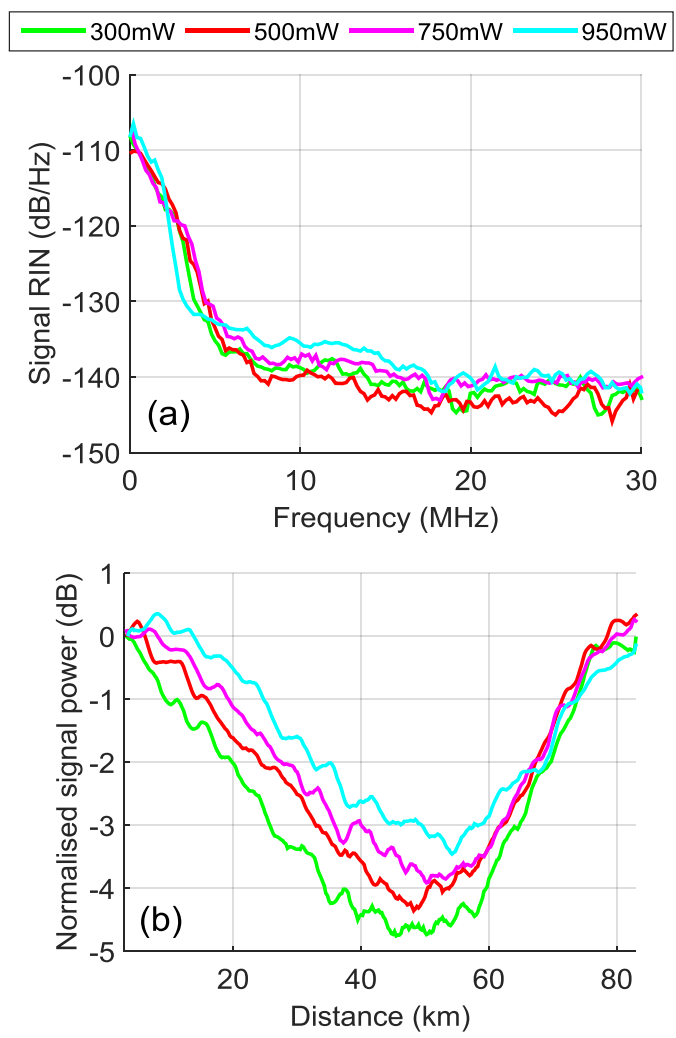

Fig. 4. (a) Signal RIN and (b) signal power variation (SPV) along the span at different forward $1365 \mathrm{~nm}$ pump powers and fixed $20 \mathrm{~mW} 1455 \mathrm{~nm}$ power.
In Fig. 4(a) and (b), the signal RIN and power profiles were characterized with different $1365 \mathrm{~nm}$ and fixed $20 \mathrm{~mW}$ broadband forward pump powers. Here in the dual order forward pumped bidirectional DRA, the improved signal power distribution and RIN transfer are mainly dominated by the high power $2^{\text {nd }}$ order forward pump. In order to ensure minimum RIN transfer from the forward $1^{\text {st }}$ order pump, we choose only $20 \mathrm{~mW}$ power of the forward $1^{\text {st }}$ order source which has much lower direct gain contribution to signal, but enough to increase the efficiency of gain transfer from forward $1365 \mathrm{~nm}$ pump to signal. Further optimization between the forward $2^{\text {nd }}$ and proposed $1^{\text {st }}$ order broadband pump powers can be done in order to find the optimum balance between the signal RIN and noise performance on long-haul transmission systems. SPVs were measured using a modified optical time domain reflectometer (OTDR) technique [22]. The forward $1365 \mathrm{~nm}$ pump powers and ratios with respect to the total pump powers are given in Table I. Signal RIN levels increased slightly below $20 \mathrm{MHz}$ and SPVs improved from $4.8 \mathrm{~dB}$ to $3 \mathrm{~dB}$ as forward $1365 \mathrm{~nm}$ pump power (ratio) was increased from $300 \mathrm{~mW}(19.4 \%)$ to $950 \mathrm{~mW}(46 \%)$. The signal power profile using dual order pumping with a broadband pump seed can be minimised to $\pm 1.5 \mathrm{~dB}$ by increasing the second order pump power, but the signal power profile in [15] can be only minimised to $\pm 2 \mathrm{~dB}$ due to the inefficient forward gain transfer. However, the absence of the first order seed in [15] mitigated the signal RIN more thoroughly.

TABLE I

FORWARD $2^{\text {ND }}$ ORDER PUMP POWER RATIOS USED IN THE CHARACTERIZATION WITH FIXED 20mW $1^{\text {ST }}$ ORDER PUMP POWER

\begin{tabular}{|c|c|c|c|}
\hline $\begin{array}{c}1365 \mathrm{~nm} \\
\text { forward pump } \\
\text { power }(\mathrm{mW})\end{array}$ & $\begin{array}{c}1365 \mathrm{~nm} \\
\text { backward pump } \\
\text { power }(\mathrm{mW})\end{array}$ & $\begin{array}{c}\text { Total pump } \\
\text { power }(\mathrm{mW})\end{array}$ & $\begin{array}{c}1365 \mathrm{~nm} \text { forward } \\
\text { pump power ratio } \\
(\%)\end{array}$ \\
\hline 300 & 1230 & 1550 & 19.4 \\
\hline 500 & 1200 & 1720 & 29.1 \\
\hline 750 & 1150 & 1920 & 39.1 \\
\hline 950 & 1096 & 2066 & 46 \\
\hline
\end{tabular}

The transmission results at different $1365 \mathrm{~nm}$ forward pump powers are also given in Fig. 5(a) and (b). The optimum balance between OSNR and RIN was achieved at $500 \mathrm{~mW}$ $(\sim 29 \%)$ of $1365 \mathrm{~nm}$ forward pump power (ratio) which
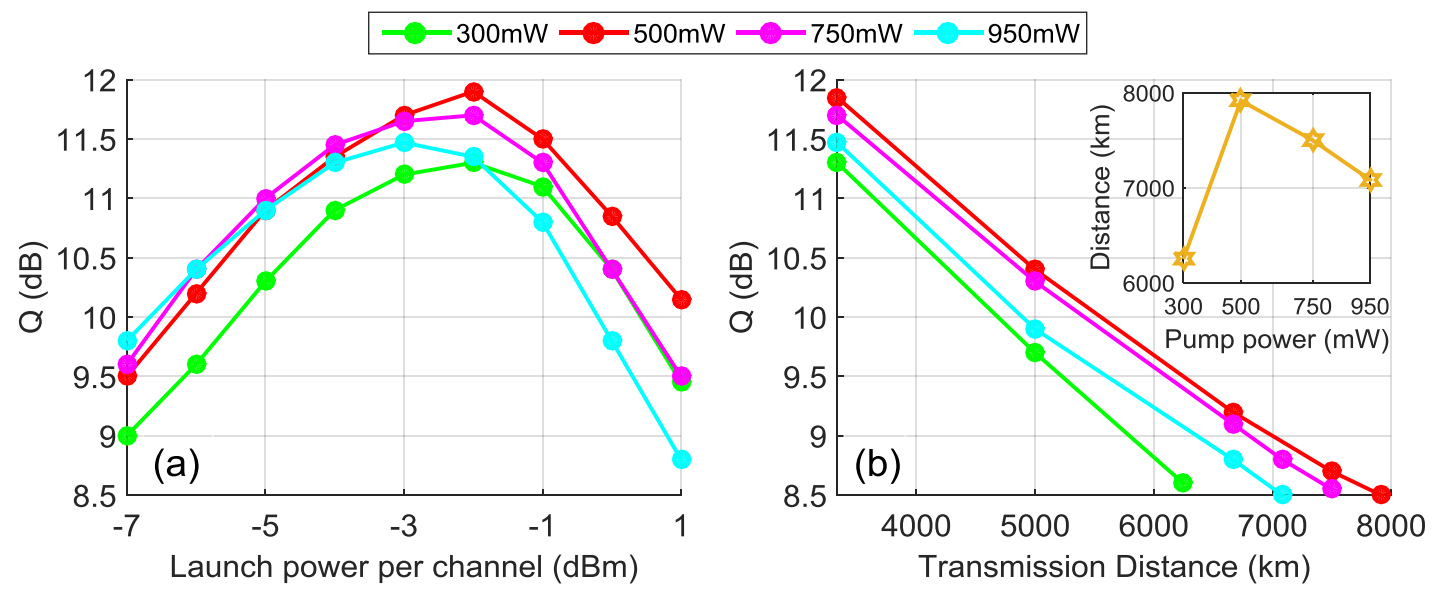

Fig. 5. Comparison of transmission performances measured at 1545.32nm signal: (a) Q factors vs. launch power per channel and (b) Q factors vs. transmission distance at optimum launch power (insert: maximum transmission distances at different $1365 \mathrm{~nm}$ forward pump powers). 
provided maximum $\mathrm{Q}$ factor $11.9 \mathrm{~dB}$ and transmission reach $7915 \mathrm{~km}$. Increasing the forward $1365 \mathrm{~nm}$ power up to $950 \mathrm{~mW}$ improved the OSNR by reducing the SPV to $3 \mathrm{~dB}$ (Fig. 4(b)) but transmission reach was degraded down to $7082 \mathrm{~km}$ due to the increase in signal RIN as shown in Fig. 4(a). On the other hand, forward $1365 \mathrm{~nm}$ power reduction to $300 \mathrm{~mW}$ resulted in poorest OSNR and lowest reach up to $6249 \mathrm{~km}$. The maximum distances at different forward $1365 \mathrm{~nm}$ pump powers are also depicted in Fig. 5(b) insert which shows the maximum reach $7915 \mathrm{~km}$ at $500 \mathrm{~mW}$, after that it deteriorates due to increased signal RIN penalty at $750 \mathrm{~mW}$ and $950 \mathrm{~mW}$. Then signal RIN, SPV characterization and transmission performances were compared with conventional $1^{\text {st }}$ order semiconductor forward pumped dual order bidirectional and backward only pumping schemes at optimized $500 \mathrm{~mW}$ and $20 \mathrm{~mW}$ forward $1365 \mathrm{~nm}$ and $1455 \mathrm{~nm}$ pump powers respectively to show the benefits.

Fig. 6 shows the comparison of signal RIN and SPV at $1545.32 \mathrm{~nm}$ among two different dual order bidirectional pumping schemes using proposed broadband and conventional low RIN semiconductor laser diode as $1^{\text {st }}$ order seed and dual order backward only pumping. In Fig. 6(a), signal RIN from backward only pumping was the lowest and baseline for minimum signal RIN. The proposed broadband pumped scheme shows similar performance as backward only pumping with slight increase in RIN level at frequencies below $6 \mathrm{MHz}$.

The $1^{\text {st }}$ order broadband seed distributes the transferred RIN from higher order noisy $1365 \mathrm{~nm}$ pump over the random phases of its wide bandwidth. The overall noise evolution to signal is then averaged out and mitigated significantly. Semiconductor forward pumped scheme shows $\sim 10 \mathrm{~dB}$ signal RIN level increase below $10 \mathrm{MHz}$ despite having the lowest pump RIN (Fig. 2(b)). In Fig. 6(b), both bidirectional pumping schemes show similar SPVs ( 4dB) with $2 \mathrm{~dB}$ improvement than backward only pumping. Our proposed dual order bidirectional DRA scheme shows improved OSNR than backward only pumping and significant signal RIN mitigation compared with conventional semiconductor pump.
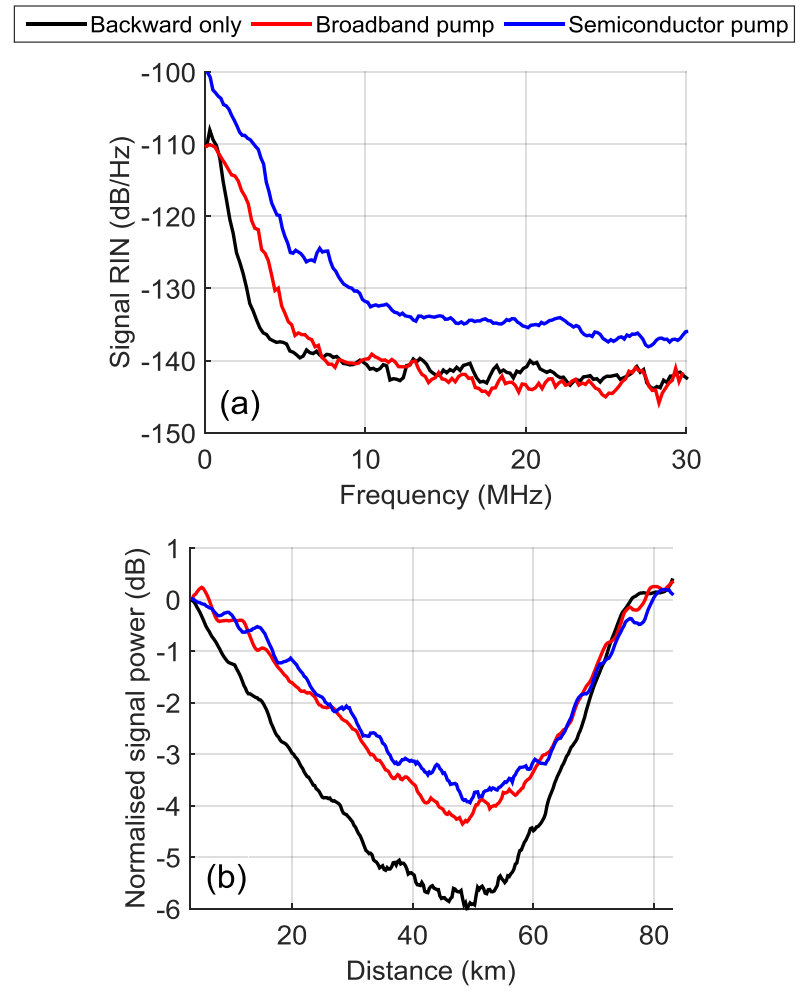

Fig. 6. Comparison of (a) signal RIN and (b) SPVs along the amplifier span for dual order forward pumped bidirectional DRAs with $1^{\text {st }}$ order broadband, semiconductor pump and backward only pumping.

Coherent WDM transmission experiments using the setup shown in Fig. 3 have been carried out at the centre WDM channel at $1545.32 \mathrm{~nm}$, in order to compare the transmission performances with backward only pumping and other bidirectional pumping schemes. In Fig. 7(a) and (b), backward only pumping shows the optimum $\mathrm{Q}$ factor of $11.5 \mathrm{~dB}$ at $3333 \mathrm{~km}$ and maximum reach of $7082 \mathrm{~km}$ respectively. Dual order forward pumping with $1^{\text {st }}$ order semiconductor pump shows $0.7 \mathrm{~dB} \mathrm{Q}$ factor penalty at optimum launch power compared with broadband $1^{\text {st }}$ order pumping due to significant signal RIN penalty. As both the dual order forward pumped DRA schemes have similar signal power profiles (Fig. 6(b)),
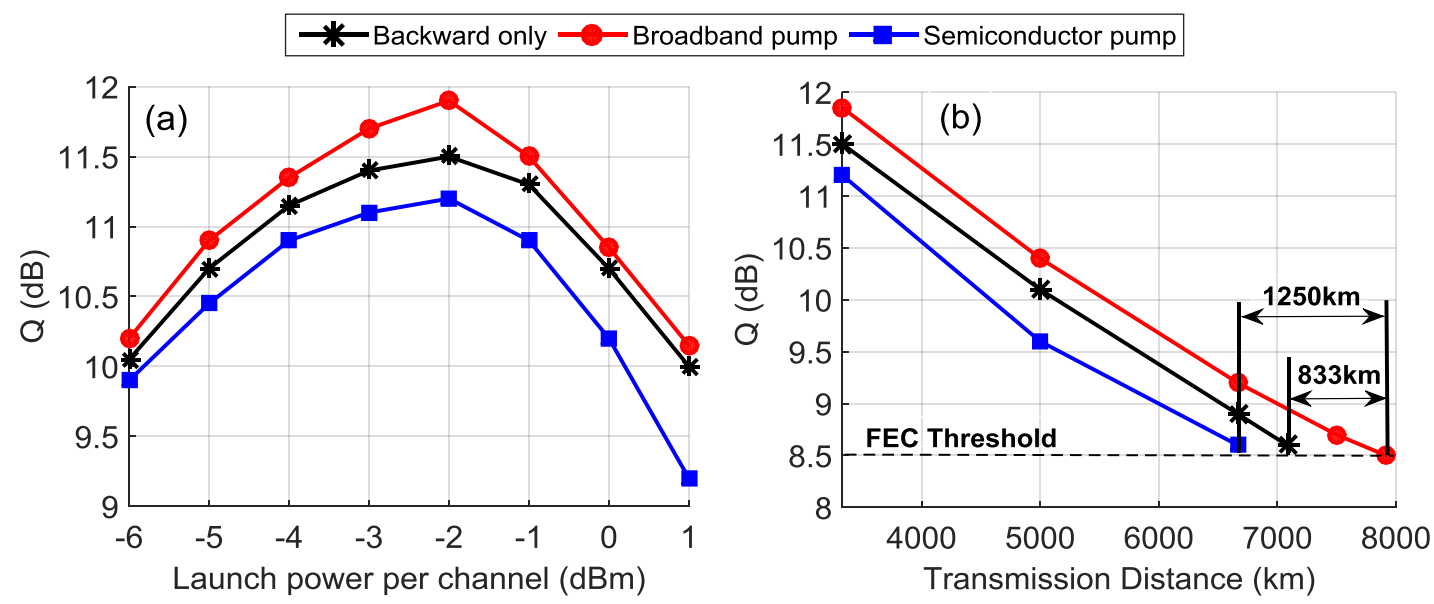

Fig. 7. Transmission performance comparison of dual order forward pumped bidirectional DRAs with different $1^{\text {st }}$ order forward pumps at fixed $500 \mathrm{~mW}$ and $20 \mathrm{~mW}$ of $2^{\text {nd }}$ and $1^{\text {st }}$ order pump powers respectively and backward only pumping: (a) Q factors vs. signal launch power per channel at $3333 \mathrm{~km}$ and (b) Q factors vs. transmission reach at optimum launch power. 
the balance between the ASE noise and nonlinear penalty is almost identical in the transmission experiment. So in the proposed broadband forward pumped bidirectional DRA, any improvement in Q factor performance comes solely from the reduction of signal RIN penalty. The benefits of reduced signal RIN and SPV with broadband $1^{\text {st }}$ order pump provided maximum $\mathrm{Q}$ factor of $11.9 \mathrm{~dB}$ and transmission reach up to $7915 \mathrm{~km}$ with $1250 \mathrm{~km}$ and $833 \mathrm{~km}$ enhanced distances compared with conventional narrowband semiconductor pump and backward only pumping respectively.

These results show that the dual order forward pumped bidirectional DRA with broadband $1^{\text {st }}$ order pump has the potential of simultaneous signal RIN mitigation and OSNR improvement. The optimum trade-off between signal RIN penalty and OSNR improvement was achieved at $500 \mathrm{~mW}$ $1365 \mathrm{~nm}$ and $20 \mathrm{~mW}$ broadband $1^{\text {st }}$ order pump powers with extended transmission reach up to $7915 \mathrm{~km}$.

\section{RIN Mitigation Using First ORder Forward PUMPING}

The RIN mitigation technique using forward pumping with only broadband $1^{\text {st }}$ order pump without the need of high power $2^{\text {nd }}$ order $1365 \mathrm{~nm}$ pump is discussed in this section. The $1^{\text {st }}$ order forward pumped DRA scheme is shown in Fig. 8, in which similar dual order backward pumping and broadband $1^{\text {st }}$ order pump generation technique are used as described in section II. Additionally, high broadband pump powers up to $250 \mathrm{~mW}$ were obtained by amplifying the generated $1455 \mathrm{~nm}$ seed from the $10 \mathrm{~km}$ SSMF through a similar $2^{\text {nd }}$ stage with $10 \mathrm{~km}$ TrueWave (TW) fibre, using the residual $1365 \mathrm{~nm}$ pump power from the first stage. The amplified broadband pump powers from the $2^{\text {nd }}$ stage at different $1365 \mathrm{~nm}$ powers are shown in Fig. 8 insert, which shows that up to $250 \mathrm{~mW}$ output power can be obtained using $\sim 3.8 \mathrm{~W}$ of $1365 \mathrm{~nm}$ pump power, beyond that the output becomes unstable due to random spikes. The amplified broadband pump has slightly narrower spectral profile than Fig. 2(a), but has equal RIN level to Fig. 2(b) for using backward pumping in the $2^{\text {nd }}$ stage.

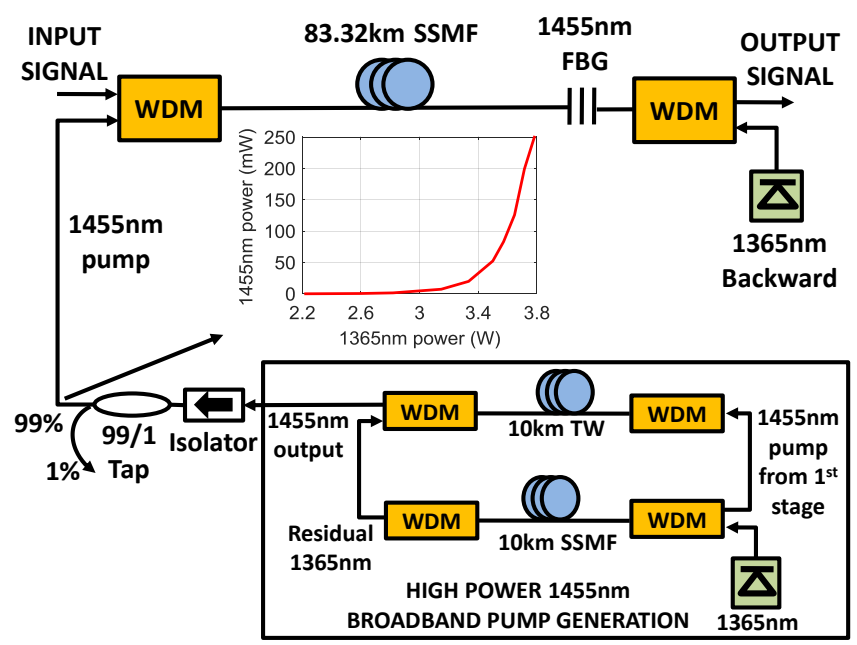

Fig. 8. First order forward pumped bidirectional DRA including the generation scheme of high power broadband $1455 \mathrm{~nm}$ pump (Insert: broadband pump power vs. generating $1365 \mathrm{~nm}$ pump powers).
The TW fibre has slightly better Raman gain efficiency $\left(0.6 \mathrm{~W}^{-1} \mathrm{~km}^{-1}\right)$ than the SSMF $\left(0.43 \mathrm{~W}^{-1} \mathrm{~km}^{-1}\right)$ and low Rayleigh scattering coefficient as SSMF. The choice of TW fibre length $(10 \mathrm{~km})$ gives a better balance between the attenuation and amplified output power. Finally, the generated broadband forward pump was given into the amplifier span through an isolator and WDM coupler. The isolator separates the seed generating section from the main amplifier span.

Full characterization of the signal RIN, power variation and transmission experiment were carried out first and then the results obtained have been compared with conventional semiconductor pump based forward pumping and dual order backward only pumping. Forward pump powers from $30 \mathrm{~mW}$ to $250 \mathrm{~mW}$ were used for measuring different SPV and RIN performances. Backward $1365 \mathrm{~nm}$ pump powers were optimized to ensure $0 \mathrm{~dB}$ signal net gain at the output of the amplifier for different forward pump powers.

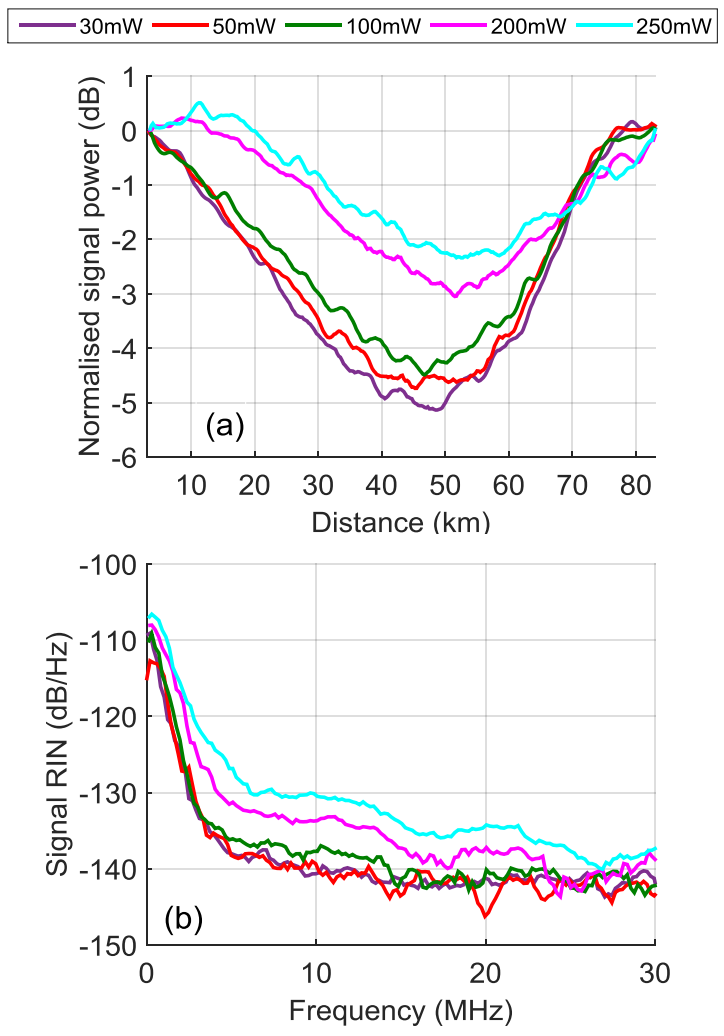

Fig. 9. Characterization of (a) signal power distributions along the amplifier span and (b) signal RIN at $1545.32 \mathrm{~nm}$ with different powers of $1^{\text {st }}$ order broadband forward pump.

The SPVs and signal RIN with increasing forward $1^{\text {st }}$ order broadband pump powers are shown in Fig. 9(a) and (b) respectively. As expected, increasing the forward pump powers from $30 \mathrm{~mW}$ to $250 \mathrm{~mW}$ reduced the SPVs from $5.1 \mathrm{~dB}$ to $2.5 \mathrm{~dB}$, resulting in improved OSNR performances. In Fig. 9(b), no significant signal RIN increase was observed up to $100 \mathrm{~mW}$ forward pump power. However a sharp increase in signal RIN level was observed beyond $100 \mathrm{~mW}$ and $10 \mathrm{~dB}$ increase in signal RIN level was also seen at $250 \mathrm{~mW}$.

The impact of improved OSNR with increasing forward pump power and associated RIN penalty have been verified 

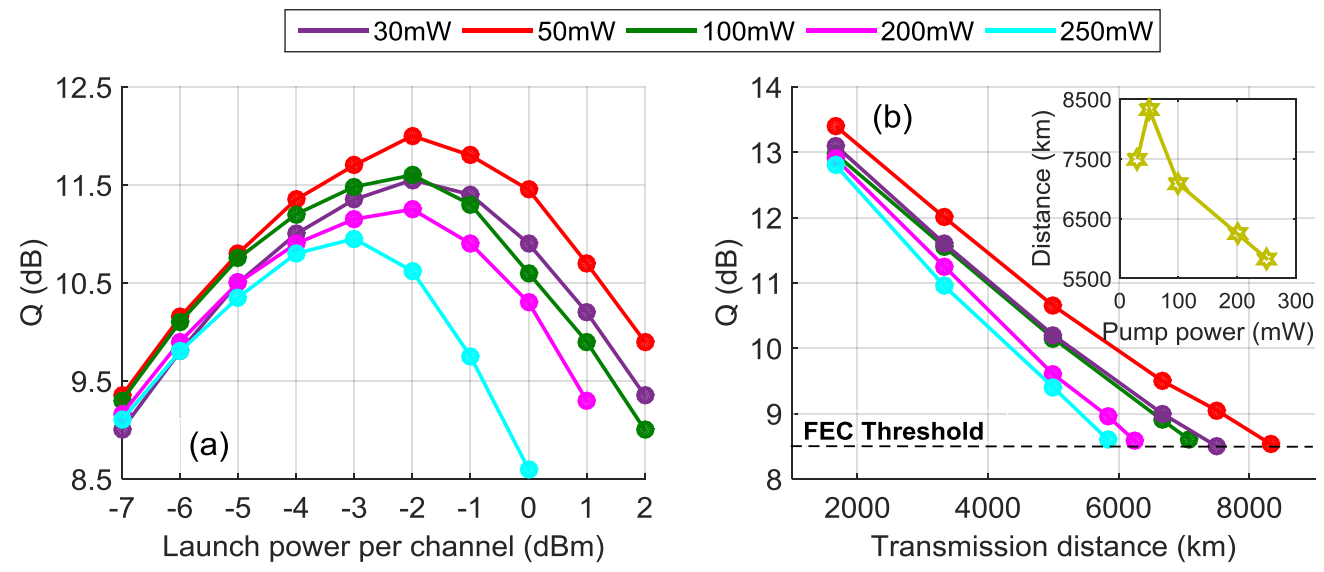

Fig. 10. Transmission performance comparison at $1545.32 \mathrm{~nm}$ signal: (a) Q factors vs. signal launch power per channel at $3333 \mathrm{~km}$ and (d) $Q$ factors vs. transmission distance with different powers of $1^{\text {st }}$ order broadband forward pump.

through transmission experiments as shown in Fig. 10(a) and (b). Maximum transmission distances were limited by signal RIN with forward pump powers above $50 \mathrm{~mW}$ as shown in Fig. 10(b) insert. Although lowest SPV (2.5dB) was achieved at $250 \mathrm{~mW}$ (Fig. 9(a)), however transmission reach was the minimum, only up to $5832 \mathrm{~km}$ with Q factor of $11 \mathrm{~dB}$ due to the high signal RIN penalty. On the other hand, signal RIN was minimum at $30 \mathrm{~mW}$ but maximum reach was limited to $7500 \mathrm{~km}$ by the lowest OSNR performance. Broadband forward pumping with $50 \mathrm{~mW}$ gave the best balance between SPV and signal RIN penalty, resulting in maximum optimum $\mathrm{Q}$ factor of $12 \mathrm{~dB}$ and maximum reach up to $8332 \mathrm{~km}$.

Signal power distributions and RIN levels were then compared with conventional $1^{\text {st }}$ order semiconductor pumped bidirectional DRA at the optimized $50 \mathrm{~mW}$ forward pump power and backward only pumping schemes as shown in Fig. 11(a) and (b) respectively. Both bidirectional DRAs showed similar SPVs with $\sim 1.3 \mathrm{~dB}$ improvement over the worst performed backward only pumping. In Fig 11(b), broadband forward pumped bidirectional DRA showed no signal RIN increase compared with the minimum RIN baseline backward only pumping. Although semiconductor pump has the lowest RIN profile (Fig. 2(b)), however a clear increase of signal RIN level was observed in semiconductor pumped scheme as shown in Fig. 11(b). This was mainly because of the coherence of narrow linewidth pump that could not average out the RIN evolution through non-degenerate FWM process.

Transmission performances were also compared with conventional bidirectional pumping using $50 \mathrm{~mW} 1^{\text {st }}$ order semiconductor pump and backward only pumping schemes as shown in Fig. 11(c) and (d). Proposed broadband pumping extended the transmission reach up to $8332 \mathrm{~km}$ with maximum
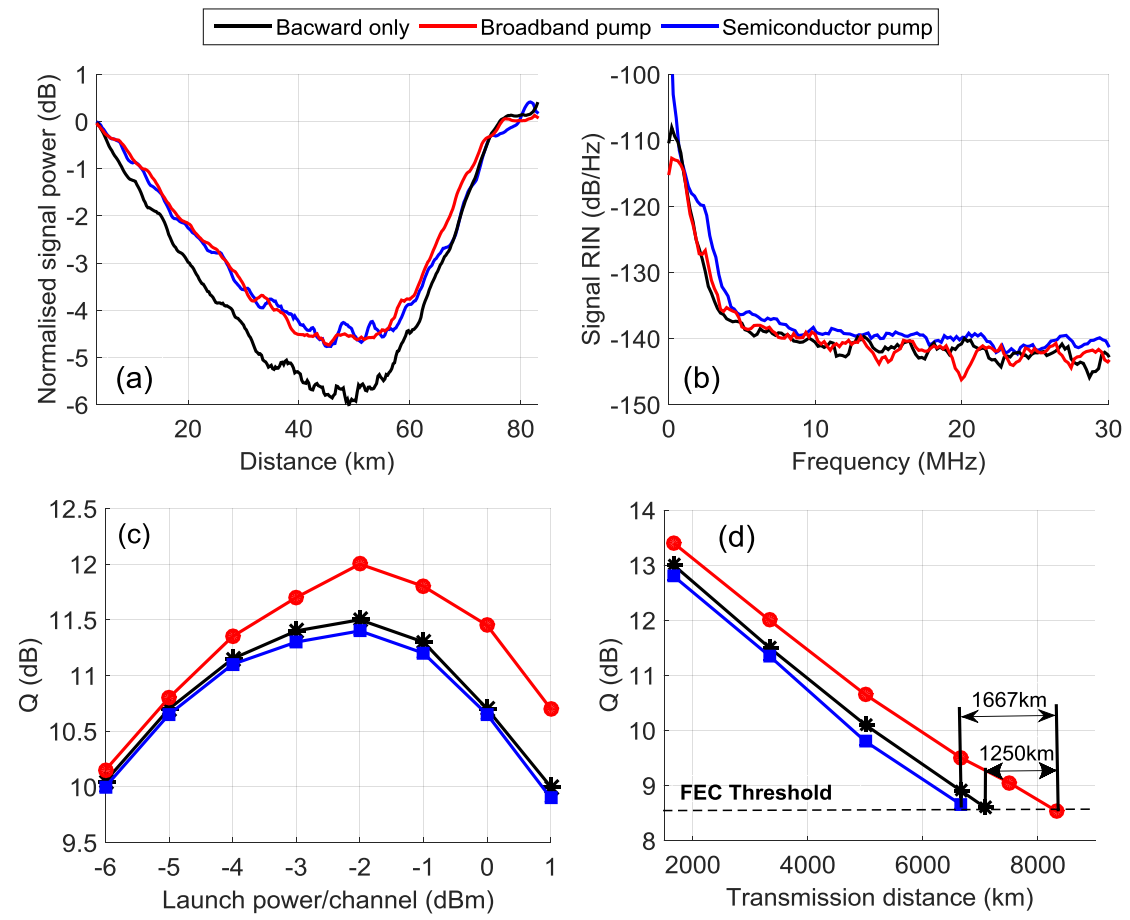

Fig. 11. Characterization (without marker) of (a) SPVs (b) signal RIN; and transmission performance comparisons (with marker) (c) Q factors vs. launch power per channel at $3333 \mathrm{~km}$ and (d) Q factors vs. transmission distance at optimum launch power. 
Q factor of $12 \mathrm{~dB}$ measured at $3333 \mathrm{~km}$. The benefit from improved OSNR by reducing the SPV resulted in minimum of $0.5 \mathrm{~dB}$ improved $\mathrm{Q}$ factor at optimum launch power than backward only pumping. Transmission distances were also enhanced by $1250 \mathrm{~km}$ and $1667 \mathrm{~km}$ compared with backward only and bidirectional pumping with forward $1^{\text {st }}$ order semiconductor pump respectively as shown in Fig. 11(d). So, first order forward pumping with reasonably low RIN broadband pump has simpler design without requiring the high power $1365 \mathrm{~nm} 2^{\text {nd }}$ order pump as in the dual order and showed the best transmission performances.

\section{CONCLUSIONS}

We have experimentally demonstrated that, a low RIN and broadband $1^{\text {st }}$ order pump can simultaneously mitigate the signal RIN and improve OSNR in both dual and $1^{\text {st }}$ order forward pumped bidirectional DRAs. In the proposed dual order forward pumped bidirectional pumping scheme, including a broadband $1^{\text {st }}$ order seed extends the transmission reach of $10 \times 120 \mathrm{~Gb} / \mathrm{s}$ DP-QPSK coherent WDM system up to maximum $7915 \mathrm{~km}$ with $0.7 \mathrm{~dB} \mathrm{Q}$ factor improvement and $1250 \mathrm{~km}$ transmission reach enhancement compared with conventional low RIN, narrowband semiconductor pump. Additionally, reach extension of $833 \mathrm{~km}$ was achieved compared with backward only pumping at optimized $1^{\text {st }}$ order broadband forward pump powers of $20 \mathrm{~mW}$ and $500 \mathrm{~mW}$ of $2^{\text {nd }}$ order $1365 \mathrm{~nm}$ pump.

Finally a simple broadband $1^{\text {st }}$ order forward pumped bidirectional DRA scheme has been demonstrated which reduces the pump power requirement. Using only $50 \mathrm{~mW}$ of broadband pump, this technique gives the best transmission performance and effectively extends the $1 \mathrm{~Tb} / \mathrm{s}$ DP-QPSK WDM transmission reach to maximum $8332 \mathrm{~km}$ with $1667 \mathrm{~km}$ and $1250 \mathrm{~km}$ reach extensions compared with $1^{\text {st }}$ order semiconductor forward pumped bidirectional and backward only pumping respectively.

Our results show that the correct choice of forward pump with low RIN (i.e. $<-135 \mathrm{~dB} / \mathrm{Hz}$ ) and most importantly very broad $3 \mathrm{~dB}$ bandwidth (i.e. $18 \mathrm{~nm}$ ) can significantly mitigate the RIN transfer from pump to signal and allow the extended reach of long-haul coherent transmission system utilizing the benefits of ASE noise reduction.

\section{REFERENCES}

[1] X. Zhou, L. E. Nelson, P. Magill, R. Isaac, B. Zhu, D. W. Peckham, P. I. Borel, and K. Carlson, "High spectral efficiency $400 \mathrm{~Gb} / \mathrm{s}$ transmission using PDM time-domain hybrid 32-64 QAM and training-assisted carrier recovery," J. Lightw. Technol., vol. 31, no. 7, pp. 999-1005, Apr. 2013.

[2] T. Omiya, M. Yoshida, and M.Nakazawa, "400 Gbit/s 256 QAMOFDM transmission over $720 \mathrm{~km}$ with a $14 \mathrm{bit} / \mathrm{s} / \mathrm{Hz}$ spectral efficiency by using high-resolution FDE," Opt. Express, vol. 21, no. 4, pp. 2632-2641, Feb. 2013.

[3] A. D. Ellis, M. Tan, Md A. Iqbal, M. A. Z. Al-Khateeb, V. Gordienko et al., " $4 \mathrm{~Tb} / \mathrm{s}$ transmission reach enhancement using $10 \times 400 \mathrm{~Gb} / \mathrm{s}$ superchannels and polarization insensitive dual band optical phase conjugation," J. Lightw. Technol., vol. 34, no. 8, pp. 1717-1723, Apr. 2016.

[4] P. Rosa, S. T. Le, G. Rizzelli, M. Tan, and J. D. Ania Castañón, "Signal power asymmetry optimisation for optical phase conjugation using
Raman amplification," Opt. Express, vol. 23, no. 25, pp. 31772-31778, Dec. 2015.

[5] S. T. Le, J. E. Prilepsky, P. Rosa, J. D. Ania-Castañón and S. K. Turitsyn, "Nonlinear inverse synthesis for optical links with distributed Raman amplification," J. Lightw. Technol., vol. 34, no. 8, pp. 17781786, Apr. 2016.

[6] S. B. Papernyi, V. I. Karpov and W. R. L. Clements, "Third-order cascaded Raman amplification," in Proc. Opt. Fiber Commun. Conf. Exhib., Mar. 2002, Paper no. FB4-1-FB4-3

[7] J. D. Ania-Castañón, "Quasi-lossless transmission using second-order Raman amplification and fibre Bragg gratings," Opt. Express, vol. 12, no. 19, pp. 4372-4377, Sept. 2004.

[8] C. R. S. Fludger, V. Handerek, and R. J. Mears, "Pump to signal RIN transfer in Raman fiber amplifiers," J. Lightw. Technol., vol. 19, no. 8, pp. 1140-1148, Aug. 2001.

[9] M. D. Mermelstein, K. Brar and C. Headley, "RIN transfer measurement and modeling in dual-order Raman fiber amplifiers," J. Lightw. Technol., vol. 21, no. 6, pp. 1518-1523, Jun. 2003.

[10] L. Xu, J. Cheng, M. Tang, J. Wu et al., "Experimental verification of relative phase noise in Raman amplified coherent optical communication system," J. Lightw. Technol., vol. 34, no. 16, pp. 3711-3716, Aug. 2016.

[11] G. Bolognini, S. Faralli, A. Chiuchiarelli, F. Falconi and F. Di Pasquale, "High-power and low-RIN lasers for advanced first- and higher order Raman copumping," IEEE Photon. Technol. Lett., vol. 18, no. 15, pp. 1591-1593, Aug. 2006.

[12] K. Keita, P. Delaye, R. Frey, and G. Roosen, "Relative intensity noise transfer of large-bandwidth pump lasers in Raman fiber amplifiers," $J$. Opt. Soc. Am. B., vol. 23, no. 12, pp. 2479-2485, Dec. 2006.

[13] M. Morimoto, H. Ogoshi, J. Yoshida, S. Takasaka, A. Sano and Y. Miyamoto, "Co-Propagating dual-order distributed Raman amplifier utilizing incoherent pumping," IEEE Photon. Technol. Lett., vol. 29, no. 7, pp. 567-570, Apr. 2017.

[14] G.Rizzelli, Md A. Iqbal, F.Gallazzi, P. Rosa, M. Tan et al., "Impact of input FBG reflectivity and forward pump power on RIN transfer in ultralong Raman laser amplifiers," Opt. Express, vol. 24, no. 25, pp. 29170-29175, Dec. 2016.

[15] M. Tan, P. Rosa, S. T. Le, Md. A. Iqbal, I. D. Phillips, and P. Harper, "Transmission performance improvement using random DFB laser based Raman amplification and bidirectional second-order pumping," Opt. Express, vol. 24, no. 3, pp. 2215-2221, Feb. 2016.

[16] M. A. Iqbal, M. Tan and P. Harper, "RIN reduction technique for dual order forward pumped distributed Raman amplification," in 2017 Conference on Lasers and Electro-Optics Europe \& European Quantum Electronics Conference (CLEO/Europe-EQEC), Jun. 2017, pp. 1-1.

[17] M. A. Iqbal, M. Tan, and P. Harper, "Evaluation of RIN mitigated dual order bidirectional distributed Raman amplification using a broadband first order forward pump," in Proc. $43^{\text {rd }}$ Eur. Conf. Opt. Commun., Sep. 2017, Paper no. P1.SC1.13.

[18] M. A. Iqbal, M. Tan, and P. Harper, "Enhanced long-haul transmission using forward propagated broadband first order Raman pump," Proc. $43^{r d}$ Eur. Conf. Opt. Commun., Sep. 2017, Paper no. P2.SC6.25.

[19] S. K. Turitsyn, S. A. Babin, D. Churkin, I. D. Vatnik, M. Nikulin, and E. V. Podivilov, "Random distributed feedback fibre lasers," Phys. Rep. vol. 542, no. 2, pp. 133-193, Sep. 2014.

[20] D. Vakhshoori et al., "Raman amplification using high-power incoherent semiconductor pump sources," in Proc. Opt. Fiber Commun. Conf. Exhib., Mar. 2003, Paper no. PD47-P1-3, vol. 3.

[21] D. V. Churkin, S. A. Babin, A. E. El-Taher, et al., "Raman Fiber Lasers with a Random Distributed Feedback Based on Rayleigh Scattering," Phys. Rev. A., vol. 82, no. 3, pp. 033828, Sep. 2010.

[22] J. D. Ania-Castanon, V. Karalekas, P. Harper, and S. K. Turitsyn, "Simultaneous spatial and spectral transparency in ultralong fiber lasers," Phys. Rev. Lett., vol. 101, pp. 123903, Sep. 2008. 